\title{
Effect of Combination Therapy of Ezetimibe and Rosuvastatin on Regression of Coronary Atherosclerosis in Patients With Coronary Artery Disease
}

\author{
Jun Masuda, ${ }^{1}$ MD, Takashi Tanigawa, ${ }^{1} \mathrm{MD}$, Tomomi Yamada, ${ }^{2} \mathrm{PhD}$, Yuki Nishimura, ${ }^{3} \mathrm{PhD}$, \\ Takashi Sasou, ${ }^{4} \mathrm{PhD}$, Tomoyuki NaKata, ${ }^{1} \mathrm{MD}$, Toshiki SawaI, ${ }^{1} \mathrm{MD}$, Naoki Fujmoto,,${ }^{1} \mathrm{MD}$, \\ Kaoru Dohi, ${ }^{1}$ MD, Masatoshi Miyahara, ${ }^{5}$ MD, Masakatsu Nishikawa, ${ }^{3}$ MD, \\ Mashio NaKAmura, ${ }^{6} \mathrm{MD}$, and Masaaki ITO, ${ }^{1} \mathrm{MD}$
}

\begin{abstract}
SUMMARY
Ezetimibe has been reported to provide significant incremental reduction in low-density-lipoprotein cholesterol (LDL-C) when added to a statin; however, its effect on coronary atherosclerosis has not yet been evaluated in detail. The aim of this study was to investigate the add-on effect of ezetimibe to a statin on coronary atherosclerosis evaluated by intravascular ultrasound (IVUS).

In this prospective randomized open-label study, a total of 51 patients with stable coronary artery disease (CAD) requiring percutaneous coronary intervention (PCI) were enrolled, and assigned to a combination group ( $n=26$, rosuvastatin $5 \mathrm{mg} /$ day + ezetimibe $10 \mathrm{mg} /$ day) or a monotherapy group $(n=25$, rosuvastatin $5 \mathrm{mg} / \mathrm{day})$. Volumetric IVUS analyses were performed at baseline and 6 months after the treatment for a non-PCI site. LDL-C level was significantly reduced in the combination group $(-55.8 \%)$ versus that in the monotherapy group $(-36.8 \% ; P=0.004)$. The percent change in plaque volume (PV), the primary endpoint, appeared to decrease more effectively in the combination group compared with the monotherapy group $(-13.2 \%$ versus $-3.1 \%$, respectively, $P=0.050)$. Moreover, there was a significant group $\times$ time interaction in the effects of the two treatments on PV $(P=0.021)$, indicating the regressive effect of the combination therapy on PV was greater than that of monotherapy for subtly different values of baseline PV in the two treatment groups. Moreover, percent change in PV showed positive correlations with percent change of LDL-C ( $r=$ $0.384, P=0.015$ ).

Intensive lipid-lowering therapy with ezetimibe added to usual-dose statin may provide significant incremental reduction in coronary plaques compared with usual-dose statin monotherapy. (Int Heart J 2015; 56: 278-285)
\end{abstract}

Key words: Cholesterol, Plaque volume, Intravascular ultrasound

$\mathrm{E}$ levated cholesterol levels are a proven risk factor for cardiovascular diseases (CVD). ${ }^{1)}$ A number of largescale clinical trials have demonstrated that cholesterollowering therapy with statins substantially reduces cardiovascular mortality and morbidity in both primary and secondary prevention. ${ }^{2,3)}$ Statins have also been shown to slow the progression or even promote the regression of coronary atherosclerosis. ${ }^{4-11)}$ Despite the established efficacy of statins, however, the attainment of low-density-lipoprotein cholesterol (LDL-C) levels recommended by the guidelines is often suboptimal. $^{12)}$

Ezetimibe is a novel cholesterol absorption inhibitor that prevents the absorption of dietary and biliary cholesterol from the small intestine. Its addition to statin therapy was well tolerated and led to further reduction in LDL-C levels by $15-$
$25 \% .^{13,14)}$ However, whether the additional LDL-C reduction achieved with the addition of ezetimibe to statin therapy leads to a further beneficial effect on coronary atherosclerosis has not been well evaluated.

In this prospective randomized open-label study, we investigated the effect of the addition of ezetimibe to statins on the regression of coronary atherosclerosis, compared with usual-dose statin monotherapy, in Japanese patients with stable coronary artery disease (CAD).

\section{MeTHODS}

Study design: This study was a prospective, open-label, randomized, single-center study designed to examine the effect of

From the ${ }^{1}$ Department of Cardiology and Nephrology, Mie University Graduate School of Medicine, Tsu, ${ }^{2}$ Department of Clinical Epidemiology and Biostatistics, Graduate School of Medicine, Osaka University, Suita, ${ }^{3}$ Clinical Research Support Center and ${ }^{4}$ Department of Clinical Engineering, Mie University Hospital, Tsu, ${ }^{5}$ Division of Cardiology, Mie Heart Center, Meiwa, and ${ }^{6}$ Department of Clinical Cardiovascular Research, Mie University Graduate School of Medicine, Tsu, Japan. Address for correspondence: Takashi Tanigawa, MD, Department of Cardiology and Nephrology, Mie University Graduate School of Medicine, 2-174 Edobashi, Tsu, Mie 514-8507, Japan. E-mail: dr.k0817@gmail.com

Received for publication September 29, 2014. Revised and accepted November 17, 2014.

Released in advance online on J-STAGE April 23, 2015.

All rights reserved by the International Heart Journal Association. 
6 months' treatment with combination therapy of ezetimibe and rosuvastatin versus usual-dose rosuvastatin monotherapy in coronary plaque regression in non-percutaneous coronary intervention (PCI) sites assessed by serial volumetric intravascular ultrasound (IVUS) analysis. Eligible patients were randomly assigned within 72 hours after a successful PCI under IVUS guidance to receive rosuvastatin at $5 \mathrm{mg}$ daily (the RSV5 group) or ezetimibe at $10 \mathrm{mg}$ and rosuvastatin at $5 \mathrm{mg}$ daily (the EZT10/RSV5 group). Follow-up visits were scheduled for 1,3, and 6 months after administration of the allocated drugs, and repeated IVUS and coronary angiography (CAG) examinations were performed at the 6-month follow-up.

The randomized treatment assignment was stratified by a minimization method controlling for the following 3 factors: 1) sex, 2) prior statin use, and 3) baseline LDL-C > $120 \mathrm{mg} / \mathrm{dL}$, using a web response system provided by a central randomization service at the Clinical Research Support Center of Mie University Hospital.

This study was conducted according to the Declaration of Helsinki and with approval from the local medical ethics committee; it was also registered at UMIN (ID: 000010323). All patients provided written informed consent.

Patient population: Patients aged 20 to 80 years with clinically stable angina pectoris were eligible to participate in the study if they were undergoing elective PCI for at least 1 significant obstructive lesion with more than $75 \%$ angiographic luminal diameter narrowing and had more than 1 untouched non-culprit target lesion for imaging with less than 50\% luminal diameter narrowing that could be clearly imaged by IVUS. Patients were also required to have an LDL-C level higher than $100 \mathrm{mg} / \mathrm{dL}(2.6 \mathrm{mmol} / \mathrm{L})$ at entry, regardless of the prior administration of statins.

Patients were excluded if they had any one of the following; a history of acute coronary syndrome within 3 months prior to study entry, heart failure (New York Heart Association class III or IV), secondary hyperlipidemia, left main CAD of more than $50 \%$ stenosis, chronic total occlusion, uncontrolled hypertension (blood pressure $>200 / 110 \mathrm{mmHg}$ despite therapy), uncontrolled diabetes (hemoglobin Alc level of $\geq 9.5 \%$ ), persistent liver dysfunction with $\geq 2 \mathrm{x}$ upper limit of normal (ULN) of serum transaminase, serum creatinine level of $>2.0$ $\mathrm{mg} / \mathrm{dL}$ or creatinine clearance of $<30 \mathrm{~mL} /$ minute, unexplained serum creatine kinase level of $>3 x$ ULN, history of allergy/ sensitivity reaction to any statin and/or ezetimibe, were already taking rosuvastatin or ezetimibe before enrollment, or were recommended for coronary artery bypass graft surgery.

IVUS examination: After PCI of the culprit lesion, baseline IVUS examination was performed. After intracoronary administration of $200 \mu \mathrm{g}$ of nitroglycerin, an IVUS catheter (2.6 Fr, $40 \mathrm{MHz}$, Atlantis SR Pro2, Boston Scientific, Marlborough, MA, USA) was advanced into the target vessel, with the transducer positioned as distal as possible to the target lesion, and withdrawn using a motorized pullback device at a speed of 0.5 $\mathrm{mm} / \mathrm{s}$. The consoles used were Galaxy 2 or iLab ultrasound systems (Boston Scientific). Images were recorded on DVD-R disks for subsequent off-line IVUS analysis. After a treatment period of 6 months, follow-up IVUS examinations of the same coronary artery segment were performed under conditions identical to those at baseline.

IVUS analysis: The target plaque lesion was defined as a nonPCI site ( $>5 \mathrm{~mm}$ proximal or distal to the PCI site, or lesions of untreated vessel) with less than 50\% angiographic luminal diameter narrowing. The analyzed segment was determined based on the following reproducible indices of IVUS images, side branches, spotty calcifications, side veins, and distances from side branch, orifice, stent edges, and bifurcation of the left anterior descending and left circumflex branches. Two independent experienced investigators who were unaware of the patient's profile and temporal sequence of paired images performed the IVUS analysis using validated planimetry software (echoPlaque3, INDEC Systems Inc., Santa Clara, CA, USA). Baseline and follow-up IVUS images were reviewed, and target segments were selected. Subsequently, every 6th frame image $(0.1 \mathrm{~mm}$ apart $)$ was measured by manual onscreen planimetry, as previously reported. ${ }^{6,7)}$ The IVUS measurements were performed according to the expert consensus on IVUS and on regression/progression studies. ${ }^{15)}$ Manual planimetry was used to trace the leading edges of the luminal and external elastic membrane (EEM) borders. To assess the reproducibility of IVUS measurements, we performed inter- and intraobserver variability analysis for measuring plaque area at exactly the same IVUS cross-sectional images. In this analysis, 63 crosssectional images from 21 patients were randomly selected and evaluated.

Calculation of efficacy measures: Coronary plaque volume (PV) was calculated as follows: $\mathrm{PV}=\Sigma\left(\mathrm{EEM}_{\mathrm{CSA}}-\mathrm{LUMEN}_{\mathrm{CSA}}\right)$, where $\mathrm{EEM}_{\mathrm{CSA}}=\mathrm{EEM}$ cross-sectional area and LU$\mathrm{MEN}_{\mathrm{CSA}}=$ luminal cross-sectional area. The primary efficacy measure, the percent change in PV during the observation period, was calculated as follows:

Percent change in PV.

The secondary efficacy measures included nominal change in percent PV (\%PV) and nominal change in normalized PV (follow-up values minus their baseline values). The $\% \mathrm{PV}$ and normalized PV were calculated as follows:

$\% \mathrm{PV}$

Normalized PV,

where L-median is the median value of the analyzed length in all subjects and L-measured is the analyzed length of each plaque. Normalized PV was calculated in order to compensate for differences in segment length between subjects and $\% \mathrm{PV}$ was calculated in order to standardize for vessel size.

Biochemical assessments: During treatment, levels of serum total cholesterol (TC), triglycerides (TG), high-density-lipoprotein cholesterol (HDL-C), and LDL-C were measured at baseline, and 1, 3, and 6 months. Levels of small dense LDL-C (sd-LDL), malondialdehyde-LDL-C (MDA-LDL), and highsensitivity C-reactive protein (hs-CRP) were measured at baseline and 6 months.

All blood samples were obtained after an overnight fast. Serum levels of TC, TG, HDL-C, and LDL-C were measured by standard enzymatic methods in our laboratory. sd-LDL was measured using a commercially available assay kit based on the precipitation method with filtration (Denka Seiken Co., Ltd., Tokyo) and MDA-LDL was measured by an enzymelinked immunosorbent assay method (Sekisui Medical Co., Ltd., Tokyo).

Statistical analysis: Continuous variables are presented as the mean (SD) in tables and as the mean (SE) in figures, and categorical variables are described using frequencies. Baseline characteristics were compared between 2 groups by an unpaired $t$-test for continuous variables and by chi-squared analy- 
sis and/or Fisher's exact test for categorical variables. A twoway repeated measures analysis of variance (ANOVA) adjusted for age was used for laboratory and IVUS data to analyze the main effect of time over the treatment periods (baseline versus follow-up), groups (the RSV5 group versus the EZT10/RSV5 group), and interaction (between group and time). As a post hoc analysis, the Bonferroni test was applied for prepost comparisons where either the main effect of time or group or an interaction achieved a $P$ value $<0.05$. Percent changes of lipid parameters and IVUS efficacy parameters were compared between groups by analysis of covariance (ANCOVA), with age and baseline variable as covariates for nominal change in normalized PV and with age as a covariate for the others. The levels of hs-CRP and TG were not distributed normally, so these data were log-transformed for statistical analysis.

We used general linear models to assess the relationships between the percent change in coronary PV and several factors, including changes of serum lipid profiles from baseline to follow-up, or to assess interobserver and intraobserver variabilities for measuring plaque area. The number of adverse events was assessed to determine safety profiles. For the sample size calculation, no information was available regarding the effect of ezetimibe combined with a statin on coronary atherosclerosis evaluated by IVUS. The addition of ezetimibe to a statin was reported to provide further reduction in LDL-C levels by $15 \%$ or more. Based on a previous IVUS study in stable Japanese CAD patients who underwent statin treatment, ${ }^{16)}$ the further reduction in percent plaque volume with ezetimibe was expected to be $8.8 \%$. Therefore, we assumed that a sample size of 21 patients per treatment group was required to detect a treatment difference of $8.8 \%$ in the primary endpoint of the percent change in PV with $80 \%$ power (assuming an SD of $10 \%$ ). With an expected drop-out of some subjects, a total of 50 patients were required. A two-sided probability value of $\leq$ 0.05 was considered significant, and all statistical analyses were performed by specialists in biostatistics at the Clinical Research Support Center of Mie University Hospital using SPSS version 20 software (SPSS, Inc., IL, USA).

\section{RESUltS}

Between October 2008 and September 2012, a total of 51 patients with stable CAD who underwent PCI at our institutes were enrolled in this study, and were randomized to the RSV5 group $(n=25)$ or the EZT10/RSV5 group $(n=26)$. A total of 40 patients had evaluable IVUS examinations at both baseline and 6-month follow-up (19 patients in the RSV5 group, 21 patients in the EZT10/RSV5 group). Of the 11 patients who were not included in the IVUS analysis, 1 did not receive the study drug; 3 were withdrawn due to an adverse event; 1 withdrew because of poor adherence; 1 did not undergo a final IVUS examination; and 3 had IVUS examinations that were not analyzable due to corruption of data. The distribution of these patients in the 2 study groups is summarized in Figure 1.

The baseline characteristics and lesion characteristics of the subjects are shown in Table I. Patients in the EZT10/RSV5 group were younger than those in the RSV5 group $(P=0.024)$. At the time of enrollment, approximately $40 \%$ of the patients were already being treated with statins. There were no signifi-

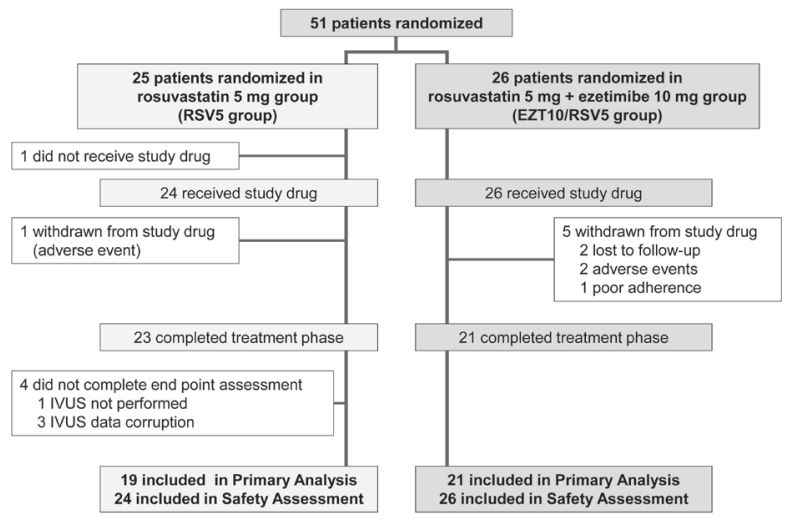

Figure 1. Flow chart of patients enrolled in the study. RSV5 indicates rosuvastatin at $5 \mathrm{mg}$ daily; and EZT10/RSV5, ezetimibe at $10 \mathrm{mg}$ plus rosuvastatin at $5 \mathrm{mg}$ daily.

Table I. Baseline Patient Characteristics

\begin{tabular}{|c|c|c|c|}
\hline Characteristic & $\begin{array}{l}\text { RSV5 group } \\
(n=19)\end{array}$ & $\begin{array}{l}\text { EZT10/RSV5 } \\
\text { group }(n=21)\end{array}$ & $P$ \\
\hline Age, years & $70.2(7.6)$ & $64.0(7.9)$ & 0.015 \\
\hline Male & $16(84.2)$ & $19(90.5)$ & 0.654 \\
\hline Body mass index, $\mathrm{kg} / \mathrm{m}^{2}$ & $23.8(2.0)$ & $24.7(4.3)$ & 0.416 \\
\hline Hypertension & $17(89.5)$ & 13 (61.9) & 0.069 \\
\hline Diabetes & $8(42.1)$ & $11(52.4)$ & 0.516 \\
\hline Family history of CAD & $6(31.6)$ & $10(47.6)$ & 0.301 \\
\hline Current smoking & $4(21.1)$ & $9(42.9)$ & 0.141 \\
\hline Previous MI & $4(21.1)$ & $5(23.8)$ & 1.000 \\
\hline Previous PCI & $5(26.3)$ & $4(19.0)$ & 0.712 \\
\hline Prior statin use & $7(36.8)$ & $9(42.9)$ & 0.698 \\
\hline \multicolumn{4}{|l|}{ Concomitant medications } \\
\hline Beta-blocker & $9(47.4)$ & $9(42.9)$ & 0.775 \\
\hline $\mathrm{ARB}$ & $10(52.6)$ & $9(42.9)$ & 0.536 \\
\hline ACE inhibitor & $3(15.8)$ & $2(9.8)$ & 0.654 \\
\hline Oral glycemic agent & $7(36.8)$ & $8(38.1)$ & 0.935 \\
\hline Insulin & $0(0.0)$ & $1(4.8)$ & 1.000 \\
\hline Type of stent used (DES) & $18(94.7)$ & $19(90.5)$ & 0.538 \\
\hline \multicolumn{4}{|l|}{ Analyzed vessel, $n$} \\
\hline RCA / LAD / LCx & $4 / 9 / 6$ & $6 / 9 / 6$ & 0.848 \\
\hline Analyzed segment & & & 0.824 \\
\hline Proximal to PCI site & $11(57.9)$ & $12(57.1)$ & \\
\hline Distal to PCI site & $6(31.6)$ & $5(23.8)$ & \\
\hline Untreated vessel & $2(10.5)$ & $4(19.0)$ & \\
\hline Lesion length, mm & $6.3(2.3)$ & $6.9(2.3)$ & 0.420 \\
\hline
\end{tabular}

Data are expressed as $n(\%)$ for categorical variables and as mean (SD) for continuous variables unless otherwise specified. ACE indicates angiotensin-converting enzyme; $\mathrm{ARB}$, angiotensin receptor blocker; CAD, coronary artery disease; DES, drug eluting stent; LAD, left anterior descending artery; LCx, left circumflex artery; MI, myocardial infarction; PCI, percutaneous coronary intervention; and RCA, right coronary artery.

cant differences between the 2 treatment groups in other patient characteristics and lesion characteristics.

Lipids and inflammatory markers: Baseline lipids were well balanced between the groups (Table II). During the 6-month treatment period, most of the lipid parameters were significantly decreased in both groups. Moreover, the degree of reduction in these parameters, except for MDA-LDL, was greater in the EZT10/RSV5 group than in the RSV5 group (interaction $P$ value $<0.05$ ). The mean percent reduction in LDL-C was significantly greater in the EZT10/RSV5 group than in the 
Table II. Baseline and Follow-up Biochemical Values

\begin{tabular}{|c|c|c|c|c|c|c|c|c|c|}
\hline & \multicolumn{3}{|c|}{$\begin{array}{l}\text { RSV5 group } \\
\quad(n=19)\end{array}$} & \multicolumn{3}{|c|}{$\begin{array}{l}\text { EZT10/RSV5 group } \\
\qquad(n=21)\end{array}$} & \multicolumn{3}{|c|}{$P$} \\
\hline & Baseline & Follow-up & \%change & Baseline & Follow-up & \%change & $\begin{array}{l}\text { Time } \\
\text { effect } P\end{array}$ & $\begin{array}{l}\text { Group } \\
\text { effect } P\end{array}$ & $\begin{array}{c}\text { Interaction } \\
\text { effect } P\end{array}$ \\
\hline Total cholesterol, mg/dL & $194.0(35.6)$ & $142.8(25.5)^{* *}$ & $-25.2(13.8)$ & $204.4(33.7)$ & $129.5(24.1)^{* *}$ & $-35.8(13.7)^{\mathbb{T I}}$ & 0.449 & 0.857 & 0.048 \\
\hline Triglycerides, mg/dL & $144.9(4.8)$ & $125.0(4.9)$ & $-4.6(15.3)$ & $129.7(5.1)$ & $84.1(5.1)$ & $-17.5(14.5)^{\mathbb{I I}}$ & 0.328 & 0.075 & 0.029 \\
\hline $\mathrm{LDL}-\mathrm{C}, \mathrm{mg} / \mathrm{dL}$ & $123.0(27.0)$ & $75.1(21.4)^{* *}$ & $-36.8(18.9)$ & $131.8(25.6)$ & $57.3(20.2)^{* *}$ & $-55.8(18.9)^{\pi}$ & 0.449 & 0.452 & 0.015 \\
\hline HDL-C, mg/dL & $47.1(12.5)$ & $49.1(16.1)$ & $4.3(19.1)$ & $53.1(11.8)$ & $57.5(15.2)$ & $8.8(19.1)$ & 0.980 & 0.101 & 0.490 \\
\hline Non-HDL-C, mg/dL & $146.2(35.6)$ & $92.8(24.7)^{* *}$ & $-34.8(17.9)$ & $151.4(29.4)$ & $74.3(23.4)^{* *}$ & $-50.3(17.9)^{\pi}$ & 0.262 & 0.360 & 0.037 \\
\hline sd-LDL, mg/dl & $29.0(9.4)$ & $18.6(8.0)^{* *}$ & $-34.4(17.0)$ & $28.1(8.3)$ & $13.0(7.1)^{* *}$ & $-53.8(16.8)^{\pi}$ & 0.763 & 0.242 & 0.037 \\
\hline MDA-LDL, U/L & $128.5(41.6)$ & $88.8(32.9)^{*}$ & $-28.6(21.6)$ & $131.8(36.9)$ & $76.8(29.1)^{* *}$ & $-38.6(21.4)$ & 0.833 & 0.688 & 0.242 \\
\hline LDL-C/HDL-C ratio & $2.7(0.7)$ & $1.6(0.6)^{* *}$ & $-38.4(19.6)$ & $2.6(0.6)$ & $1.1(0.6)^{* *}$ & $-58.2(19.5)^{\mathbb{\pi}}$ & 0.131 & 0.088 & 0.043 \\
\hline hs-CRP, mg/dL & $0.077(0.006)$ & $0.034(0.006)$ & $-14.4(30.4)$ & $0.092(0.006)$ & $0.037(0.006)$ & $-18.8(28.7)$ & 0.144 & 0.797 & 0.764 \\
\hline Hemoglobin A1c, \% & $6.5(1.0)$ & $6.6(1.3)$ & $2.3(9.9)$ & $6.4(0.9)$ & $6.6(1.2)$ & $2.6(9.8)$ & 0.669 & 0.965 & 0.898 \\
\hline
\end{tabular}

All values are presented as mean and (SD). To convert the values for cholesterol to mmol/L, multiply by 0.0259 . To convert triglycerides to mmol/L, multiply by $0.0113 .{ }^{*} P<0.05$ versus baseline, ${ }^{* *} P<0.01$ versus baseline. ${ }^{\pi} P<0.05$ for EZT10/RSV5 group versus RSV5 group, calculated with the use of analysis of covariance, with age as a covariate. LDL-C indicates low-density-lipoprotein cholesterol; HDL-C, high-density-lipoprotein cholesterol; Non-HDL$\mathrm{C}$, non-high-density-lipoprotein cholesterol; sd-LDL, small dense LDL; MDA-LDL, malondialdehyde LDL; and hs-CRP, high-sensitivity C-reactive protein.

Table III. Baseline and Follow-Up Intravascular Ultrasound Results

\begin{tabular}{|c|c|c|c|c|c|c|c|}
\hline & \multicolumn{2}{|c|}{$\begin{array}{l}\text { RSV5 group } \\
\quad(n=19)\end{array}$} & \multicolumn{2}{|c|}{$\begin{array}{l}\text { EZT10/RSV5 group } \\
(n=21)\end{array}$} & \multicolumn{3}{|c|}{$P$} \\
\hline & Baseline & Follow-up & Baseline & Follow-up & $\begin{array}{c}\text { Time effect } \\
P\end{array}$ & $\begin{array}{c}\text { Group effect } \\
P\end{array}$ & $\begin{array}{c}\text { Interaction } \\
\text { effect } P\end{array}$ \\
\hline Lesion length, mm & $6.3(2.3)$ & $6.3(2.3)$ & $6.9(2.3)$ & $6.9(2.3)$ & 0.444 & 0.536 & 0.787 \\
\hline EEM volume, $\mathrm{mm}^{3}$ & $95.0(53.8)$ & $90.5(48.7)$ & $105.9(53.6)$ & $101.9(48.5)$ & 0.500 & 0.509 & 0.877 \\
\hline Lumen volume, $\mathrm{mm}^{3}$ & $51.5(31.8)$ & $49.6(29.8)$ & $50.5(31.7)$ & $54.8(29.7)$ & 0.739 & 0.830 & 0.068 \\
\hline $\mathrm{PV}, \mathrm{mm}^{3}$ & $43.5(28.5)$ & $40.9(24.7)$ & $55.3(28.4)$ & $47.1(24.6)$ & 0.584 & 0.307 & 0.021 \\
\hline Normalized PV, $\mathrm{mm}^{3}$ & $42.0(19.2)$ & $40.1(16.5)$ & $47.3(19.1)$ & $40.5(16.4)^{*}$ & 0.250 & 0.625 & 0.030 \\
\hline Percent PV, \% & $46.4(12.1)$ & $45.7(12.6)$ & $52.5(12.1)$ & $46.9(12.6)$ & 0.386 & 0.359 & 0.028 \\
\hline
\end{tabular}

Values are expressed as mean (SD). Data were analyzed by two-way repeated measures analysis of variance after adjustment for age followed by post hoc comparisons. ${ }^{*} P<0.05$ versus baseline. EEM indicates external elastic membrane; and PV, plaque volume.

RSV5 group $(-55.8 \%$ versus $-36.8 \%, P=0.004)$. The mean percent reductions in TG and non-HDL-C were also greater in the EZT10/RSV5 group than in the RSV5 group $(-31.4 \%$ versus $-8.2 \%, P=0.021 ;-50.3 \%$ versus $-34.8 \%, P=0.012$, respectively). Similarly, the mean percent reduction in sd-LDL was also significantly greater in the EZT10/RSV5 group than in the RSV5 group $(-53.8 \%$ versus $-34.4 \%, P=0.002)$. The inflammatory marker hs-CRP did not change in either group during the treatment period.

IVUS results: Baseline and follow-up IVUS results analyzed by two-way repeated measures ANOVA after adjustment for age are shown in Table III, and the results of primary and secondary efficacy parameters are shown in Figure 2. There was no statistically significant difference in the baseline EEM volume $(P=0.541)$, lumen volume $(P=0.886)$, plaque volume $(P$ $=0.216)$, or normalized PV $(P=0.407)$ between the two treatment groups. The percent change in PV, the primary efficacy parameter, appeared to decrease more effectively in the EZT10/RSV5 group compared with that in the RSV5 group, but this difference failed to reach statistical significance $(-13.2 \%$ in the combination group and $-3.1 \%$ in the monotherapy group, $P=0.050$, Figure 2 ). However, there was a significant group $\times$ time interaction in the effects of the two treatments on the PV $(P=0.021$, Table III), indicating the regressive effect of the combination therapy on PV was greater than that of monotherapy for different values of baseline PV in

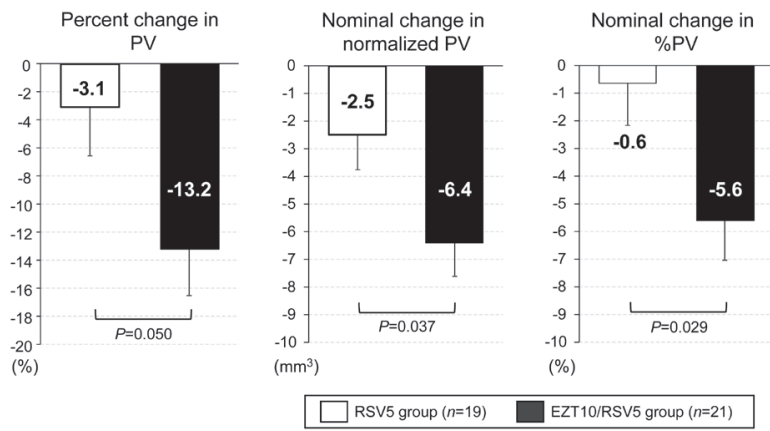

Figure 2. Results of primary and secondary efficacy parameters. The percent change in plaque volume was significantly lower in the EZT10/RSV5 group than in the RSV5 group. Moreover, nominal change in normalized PV and nominal change in percent PV were also significantly lower in the EZT10/RSV5 group. PV indicates plaque volume; and \%PV, percent plaque volume.

the two treatment groups. In the EZT10/RSV5 group, normalized PV significantly decreased during the study period (47.3 $\pm 18.2 \mathrm{~mm}^{3}$ versus $\left.40.5 \pm 16.4 \mathrm{~mm}^{3}, P<0.001\right)$, while it was not affected in the RSV5 group (time effect, $P=0.25$; group effect, $P=0.625$; interaction effect, $P=0.03$ ). In terms of the percent $\mathrm{PV}$, the interaction effect $P$ value was also significant $(P=0.028)$, although the change of the percent PV was not 
statistically significant in the EZT10/RSV5 group.

Nominal change in normalized PV, a secondary efficacy parameter, showed a significant favorable effect in the EZT10/ RSV5 group with a mean reduction of $6.4 \mathrm{~mm}^{3}$ compared with a reduction of $2.5 \mathrm{~mm}^{3}$ in the RSV5 group $(P=0.037$ for the between-group comparison) (Figure 2). A similar difference was also observed for nominal change in $\% \mathrm{PV}$, with a reduction of 5.6\% in the EZT10/RSV5 group and $0.6 \%$ in the RSV5 group ( $P=0.029$ between groups). There were no significant differences in the percent change in PV between the different analyzed segments; proximal to PCI site, distal to PCI site and untreated vessel (ANOVA $P \geqq 0.62$ for each treatment group). Figure 3 shows representative examples of IVUS in a single patient at baseline and the follow-up period in the EZT10/ RSV5 group.

Figure 4 shows correlations between the percent change in PV and lipid parameters. Significant correlations between the percent change in PV and the lipid parameters were observed: $r=0.384(P=0.015)$ for the percent change in PV and the percent change in LDL-C, and $r=0.356(P=0.033)$ for the percent change in PV and the follow-up sd-LDL level. The percent change in PV was also positively correlated with the percent change in non-HDL-C $(r=0.334, P=0.035)$. There was no significant difference in the association of percent LDL-C reduction and \%PV reduction between the two treatment groups (data not shown).

Intraobserver and interobserver variability: The correlation coefficient and mean difference \pm SD were 0.991 and $-0.09 \pm$ $0.33 \mathrm{~mm}^{2}$ (of the absolute mean value, $3.31 \pm 2.35 \mathrm{~mm}^{2}$, of the samples) for intraobserver variability and 0.990 and $-0.09 \pm$ $0.34 \mathrm{~mm}^{2}\left(2.90 \pm 2.05 \mathrm{~mm}^{2}\right)$ for interobserver variability, respectively.
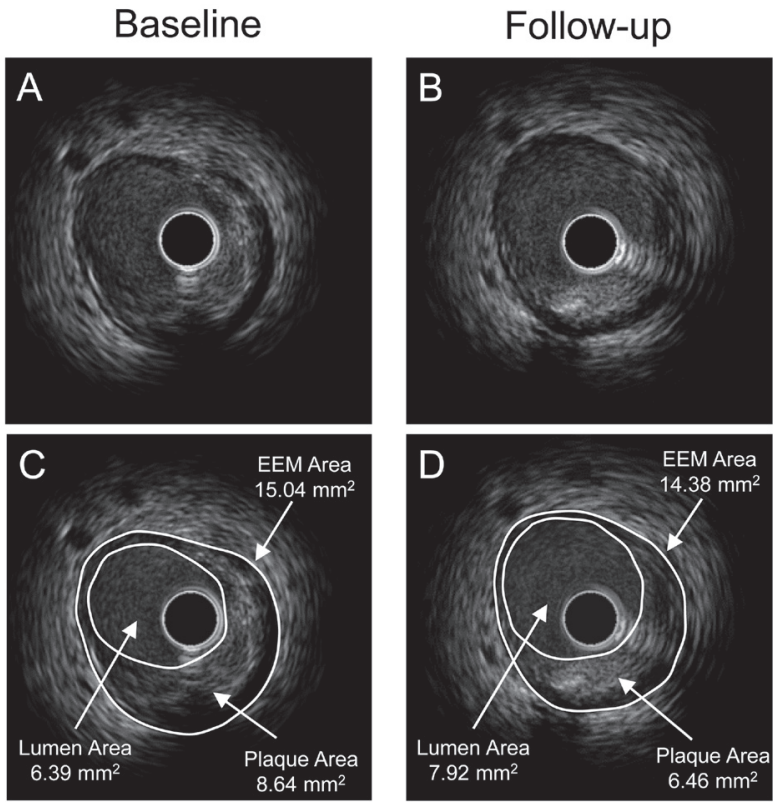

Figure 3. Representative images of intravascular ultrasound (IVUS) from a case in the EZT10/RSV5 group. A: A single cross-sectional IVUS image at baseline. B: The same cross section at follow-up. C, D: The bottom 2 panels illustrate the same cross section with measurements superimposed. A significant reduction in plaque area and increase in lumen area were observed after 6 months.
Adverse events: Table IV shows the adverse events (AE) recorded during the study period. Adverse drug reactions occurred in 2 subjects: 1 instance of drug eruption in the EZT10/ RSV5 group and 1 instance of myalgia in the RSV5 group. The rates of laboratory abnormality were similar in the two groups.

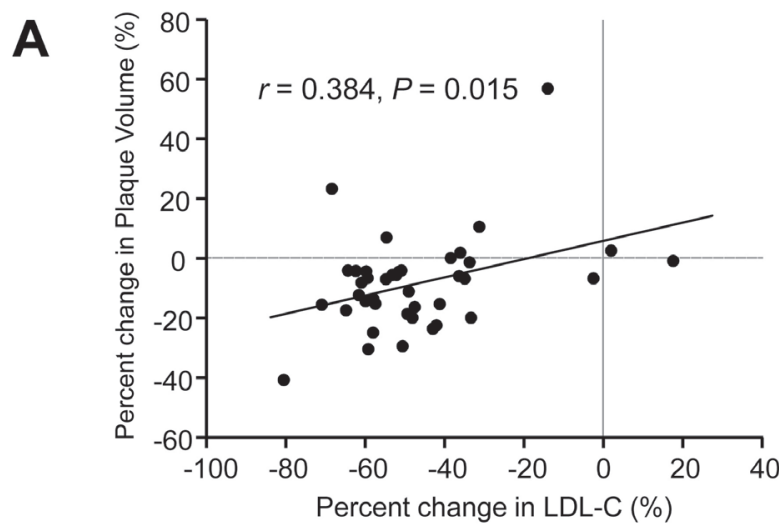

B
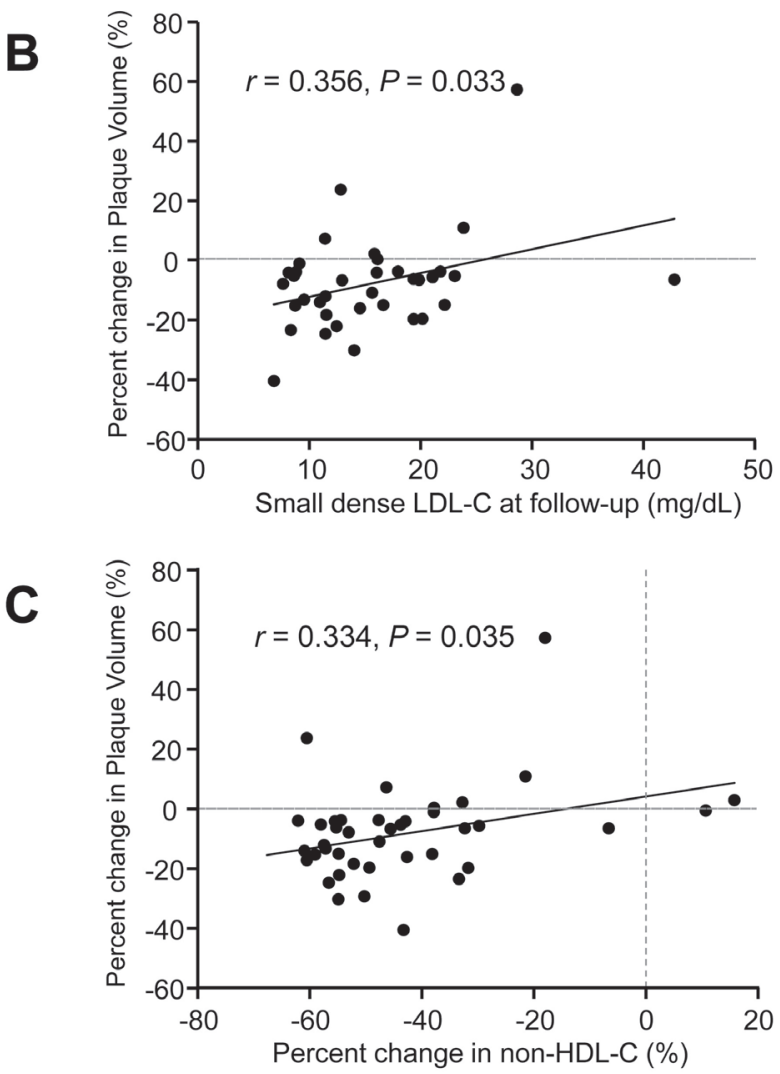

Figure 4. Relationship between lipid parameters and percent change in plaque volume during 6-month follow-up. A: Relationship between the percent change in LDL-C and percent change in plaque volume. B: Relationship between the follow-up small dense LDL-C level and percent change in plaque volume. C: Relationship between the percent change in non-HDL-C and percent change in plaque volume. LDL-C indicates lowdensity-lipoprotein cholesterol; and non-HDL-C, non-high-density-lipoprotein cholesterol. 
Table IV. Adverse Events and Drug Discontinuation

\begin{tabular}{|c|c|c|c|}
\hline & $n$ & $\begin{array}{l}\text { RSV5 } \\
\text { group }\end{array}$ & $\begin{array}{c}\text { EZT10/RSV5 } \\
\text { group }\end{array}$ \\
\hline \multicolumn{4}{|l|}{ Major adverse events } \\
\hline Myocardial infarction & 0 & $0(0.0)$ & $0(0.0)$ \\
\hline Death & 0 & $0(0.0)$ & $0(0.0)$ \\
\hline Stroke & 0 & $0(0.0)$ & $0(0.0)$ \\
\hline Rhabdomyolysis & 0 & $0(0.0)$ & $0(0.0)$ \\
\hline Coronary revascularization (TLR) & 2 & $1(4.2)$ & $1(3.8)$ \\
\hline \multicolumn{4}{|l|}{ Abnormality of laboratory value } \\
\hline AST or ALT $>3 \times$ ULN & 0 & $0(0.0)$ & $0(0.0)$ \\
\hline Creatine kinase $(\mathrm{CK})>5 \times \mathrm{ULN}$ & 2 & $1(4.2)$ & $1(3.8)$ \\
\hline \multicolumn{4}{|l|}{ Adverse drug reaction } \\
\hline Myalgia & 1 & $1(4.2)$ & $0(0.0)$ \\
\hline Drug eruption & 1 & $0(0.0)$ & $1(3.8)$ \\
\hline \multicolumn{4}{|c|}{ Discontinuation of treatment due to adverse event } \\
\hline Drug-related adverse event & 2 & $1(4.2)$ & $1(3.8)$ \\
\hline Other (renal failure due to CCE) & 1 & $0(0.0)$ & $1(3.8)$ \\
\hline
\end{tabular}

Data are expressed as $n(\%)$. ALT indicates alanine aminotransferase; AST, aspartate aminotransferase; CCE, cholesterol crystal embolism; TLR, target lesion revascularization; and ULN, upper limit of normal.

\section{Discussion}

The present study demonstrated that the addition of ezetimibe to usual-dose rosuvastatin induced a significantly greater reduction in coronary PV than usual-dose rosuvastatin monotherapy in patients with stable CAD. Moreover, the degree of coronary PV reduction was not only positively correlated with the percent LDL-C reduction, but also with the percent non-HDL-C reduction, and with the levels of sd-LDL at follow-up. To the best of our knowledge, this is the first randomized controlled IVUS study to evaluate the additional effect of ezetimibe on top of usual-dose statin on coronary atherosclerosis.

Lipid lowering effect of ezetimibe: The additional benefit of ezetimibe added to a statin on atherosclerosis progression has not been well elucidated, in spite of its additional powerful LDL-C-lowering effect. In several previous studies, it was reported that treatment with ezetimibe alone or in combination with a statin did not alter LDL particle size, but altered the LDL subfraction distribution towards increased concentrations of atherogenic small dense particles, which resulted in a more atherogenic LDL subfraction profile. ${ }^{17)}$ However, most of the recent papers reported favorable effects for ezetimibe on sdLDL. In particular, combination therapy with ezetimibe and a statin provided a significant reduction in sd-LDL of up to $46 \%{ }^{18)}$ This is comparable to our result in the combination group $(50.6 \%$ reduction in sd-LDL). Although conclusive evidence of the clinical significance of sd-LDL is still lacking, the predominance of sd-LDL has been highly associated with increased risk for $\mathrm{CAD},{ }^{19)}$ and accepted as an emerging cardiovascular risk factor by the current guidelines of the third National Cholesterol Education Program-Adult Treatment Panel (NCEP-ATP III). ${ }^{20)}$ On the other hand, there is accumulating evidence that non-HDL-C is a better predictor of CVD risk than LDL-C levels. ${ }^{21)}$ In the present study, compared with rosuvastatin alone, combination therapy significantly decreased not only LDL-C but also other lipid components, such as TG, non-HDL-C, and sd-LDL. These reductions in atherogenic lipid components may have led to the favorable effect on coronary atherosclerosis in the combination group. In fact, the de- gree of coronary PV reduction was positively correlated with the percent reduction in LDL-C and non-HDL-C, and with the levels of sd-LDL at follow-up.

Comparison with previous clinical trials of ezetimibe: To date, no large randomized trial has shown a significant reduction in clinical events with combination therapy with ezetimibe and a statin versus statin alone. Therefore, there has been significant controversy regarding the clinical effectiveness of ezetimibe. In the ENHANCE study, contrary to our result, the addition of ezetimibe to simvastatin did not significantly change carotid intima media thickness (cIMT). ${ }^{22)}$ This discrepancy may be due to several differences in the target populations, including a higher prevalence of prior statin therapy (almost $80 \%$ of patients in the ENHANCE study compared with almost $40 \%$ in our study) and a lower rate of established CAD in the ENHANCE study. In contrast to the ENHANCE study, some previous studies reported the beneficial effect of combination therapy with ezetimibe and a statin on carotid atherosclerosis, consistent with our results. ${ }^{23,24)}$ More recently, there have been several studies that supported the antiatherosclerotic effect of ezetimibe. Habara, et al reported that combination therapy with ezetimibe and fluvastatin increased the fibrous cap thickness of lipid-rich coronary plaque compared with fluvastatin alone in patients with stable CAD. ${ }^{25)}$ Bogiatzt, et al reported in their observational study that the addition of ezetimibe to ongoing statin administration produced significant regression of carotid plaque burden. ${ }^{26}$

Comparison with previous IVUS studies: The present reduction in atheroma volume was considerably larger than those previously reported in Western populations, such as in ASTEROID and REVERSAL. ${ }^{4,5)}$ One of the potential reasons for this is the differences in IVUS methodology. In those trials, IVUS measurement was performed on cross-sectional images obtained at $1.0-\mathrm{mm}$ intervals, whereas in our study, the separation between cross-sectional images was $0.1 \mathrm{~mm}$. Therefore, the previous studies showed the general effect of statins on longer coronary segments, whereas we evaluated more specific effects on local plaque segments. Indeed, the plaques in the most diseased 10-mm segments showed more regression than the entire coronary artery vasculature in those trials. The de- 
gree of percent change in PV in our study for the combination group $(-13.2 \%)$ was similar to that reported in previous investigations in Japanese patients with CAD by the ESTABLISH study $(-13.1 \%$ for the atorvastatin group) and by the JAPANACS study (-17.5\% for total patients), although some differences in the target populations and evaluation methodology preclude direct comparison with our study. ${ }^{7,8)}$ It is also possible that there are genetic or ethnic differences between Japanese patients with CAD and similar Caucasian subjects in terms of the response to lipid-lowering therapy. The present results of percent reduction in LDL-C and degree of plaque regression in the rosuvastatin group ( $5 \mathrm{mg} /$ day) are comparable to the results from the intensive atorvastatin group $(80 \mathrm{mg} /$ day $)$ in the SATURN trial, which found about a $41.5 \%$ reduction in LDL-C and a $0.99 \%$ reduction in PAV in the atorvastatin group. ${ }^{9}$

Recently, similarly to our findings, Nakajima, et al reported that additional LDL-C reduction with ezetimibe and a statin provided greater plaque regression with borderline statistical significance $(P=0.06)$ in patients with ACS. ${ }^{27)}$ Notably, they did not examine the group $\times$ time interaction effect for the comparison of the two treatments on PV reduction in spite of the subtle differences in baseline values of PV between the two groups. In addition, their study was not a prospective randomized study and therefore did not directly compare PV between the two treatment groups. Moreover, the target population in their study was different from that in the present study.

Comparison with intensive statin therapy: Previous clinical IVUS trials have reported that intensive statin therapy resulted in a greater reduction in coronary atherosclerosis compared with moderate-dose statin therapy. For example, intensive treatment with rosuvastatin for stable CAD has been reported to provide a significant reduction of plaque volume by 4.2 to $6.8 \%$ in trials from western countries, ${ }^{5,9)}$ and by $5.1 \%$ in a Japanese trial. ${ }^{6}$ On the other hand, the present result of combination therapy with ezetimibe, a reduction in plaque volume by $13.2 \%$, was considerably larger than these previous results of intensive statin treatment. These findings suggest that the effect of combination therapy with ezetimibe on coronary atherosclerosis might be equivalent to or more beneficial than that of intensive statin monotherapy. However, it was difficult to compare directly the present regressive effect in the combination therapy to those in the previous studies treated with intensive statin alone, because there were several differences in the target populations, attained LDL-C levels, and IVUS methodology. Further studies are needed to evaluate whether the effects of the combination therapy with ezetimibe on coronary atherosclerosis are greater than those of intensive statin therapy with similar LDL-C reduction.

Clinical implication: We believe that the current study has important implications for the efficacy of ezetimibe and optimal treatment of CAD. The current study results suggest that the incremental reduction of atherogenic lipoproteins achieved with ezetimibe on top of statin administration could lead to further regression in coronary atherosclerosis.

Although statin therapy is the mainstay for LDL-C lowering, attainment of the recommended target of LDL-C by a statin alone is often sub-optimal. Several recent studies have shown that $40-80 \%$ of high risk patients treated with statins could not achieve the recommended target of LDL-C. ${ }^{12)}$ Recent reports indicate that high-dose statins may be associated with an increased incidence of side effects including elevated hepat- ic transaminase, myopathy, and new-onset diabetes mellitus. ${ }^{28,29)}$ For these reasons, addition of ezetimibe to usual-dose statins appears to be a practical and effective therapeutic option for patients with $\mathrm{CAD}$ who are either unable to achieve the LDL-C target with a statin alone or are at increased risk for side effects with more potent doses of statin.

Study limitations: In terms of the limitations of this study, first, this single-center study enrolled only a small number of subjects, which limited clinical interpretation. In the present study, the statistical power was 0.523 with type I error of 0.05 and a treatment difference between groups of $10.1 \%$ (SD of $15.3 \%$ ). When we re-calculated sample size based on our results, we found we would need 37 patients per treatment group. Therefore, these data should be regarded as preliminary because of the small sample size. Additional larger prospective randomized studies with a long-term outcome are needed to confirm our findings. Second, the follow-up period was relatively short, which limits the conclusions that can be made regarding longer-term therapy. Longer follow-up may be necessary to evaluate the impact of the combination therapy on coronary atherosclerosis and adverse cardiac events. We also performed another follow-up CAG at 12 to 18 months after PCI, which resulted in no significant difference in the rate of coronary restenosis between the two groups. Third, this study only assessed local plaques in part of the coronary arteries. Arbitrary short segment analysis might not necessarily provide representative results for the entire population of coronary arteries. Fourth, age was not included into the factors for randomization due to the small number of subjects. As there was a significant difference in age between groups, all laboratory and IVUS data were analyzed after adjustment for age. Finally, patients already administered statins, as well as statin-naïve patients, were included without a wash-out period in this study because these criteria were believed useful to represent actual clinical practice.

Conclusions: The combination therapy with ezetimibe and a usual-dose statin provided significant incremental reduction in coronary plaques compared with usual-dose statin monotherapy in patients with stable CAD. Our results might suggest that the additional reduction of atherogenic lipoproteins achieved with ezetimibe on top of a statin translates into further regression in coronary atherosclerosis.

\section{ACKNOWLEDGMENT}

The authors thank Dr Satoshi Tamaru for his important contributions.

\section{Disclosure}

Conflict of interest: Mie University Graduate School of Medicine received minor unrestricted research grants from MSD, AstraZeneca, and Shionogi Co. Ltd. No relevant conflicts of interest were disclosed by the authors.

\section{REFERENCES}

1. Lewington S, Whitlock G, Clarke R, et al. Blood cholesterol and 
vascular mortality by age, sex, and blood pressure: a meta-analysis of individual data from 61 prospective studies with 55,000 vascular deaths. Lancet 2007; 370: 1829-39. (Review)

2. Baigent C, Blackwell L, Emberson J, et al. Efficacy and safety of more intensive lowering of LDL cholesterol: a meta-analysis of data from 170,000 participants in 26 randomised trials. Lancet 2010; 376: 1670-81.

3. Mills EJ, Rachlis B, Wu P, Devereaux PJ, Arora P, Perri D. Primary prevention of cardiovascular mortality and events with statin treatments: a network meta-analysis involving more than 65,000 patients. J Am Coll Cardiol 2008; 52: 1769-81.

4. Nissen SE, Tuzcu EM, Schoenhagen P, et al. Effect of intensive compared with moderate lipid-lowering therapy on progression of coronary atherosclerosis: a randomized controlled trial. JAMA 2004; 291: 1071-80.

5. Nissen SE, Nicholls SJ, Sipahi I, et al. Effect of very high-intensity statin therapy on regression of coronary atherosclerosis: the ASTEROID trial. JAMA 2006; 295: 1556-65.

6. Takayama T, Hiro T, Yamagishi M, et al. Effect of rosuvastatin on coronary atheroma in stable coronary artery disease: multicenter coronary atherosclerosis study measuring effects of rosuvastatin using intravascular ultrasound in Japanese subjects (COSMOS). Circ J 2009; 73: 2110-7.

7. Hiro T, Kimura T, Morimoto T, et al. Effect of intensive statin therapy on regression of coronary atherosclerosis in patients with acute coronary syndrome: a multicenter randomized trial evaluated by volumetric intravascular ultrasound using pitavastatin versus atorvastatin (JAPAN-ACS [Japan assessment of pitavastatin and atorvastatin in acute coronary syndrome] study). J Am Coll Cardiol 2009; 54: 293-302.

8. Okazaki S, Yokoyama T, Miyauchi K, et al. Early statin treatment in patients with acute coronary syndrome: demonstration of the beneficial effect on atherosclerotic lesions by serial volumetric intravascular ultrasound analysis during half a year after coronary event: the ESTABLISH Study. Circulation 2004; 110: 1061-8.

9. Nicholls SJ, Ballantyne CM, Barter PJ, et al. Effect of two intensive statin regimens on progression of coronary disease. $\mathrm{N}$ Engl J Med 2011; 365: 2078-87.

10. Kaneko H, Yajima J, Oikawa Y, et al. Recent characteristics and outcomes of Japanese stable angina pectoris after percutaneus coronary intervension. An observational cohort study using the Shinken Database. Int Heart J 2013; 54: 335-40.

11. Yokoi H, Nohara R, Daida $\mathrm{H}$, et al. Change in carotid intima-media thickness in a high-risk group of patients by intensive lipidlowering therapy with rosuvastatin : subanalysis of the JART study. Int Heart J 2014; 55: 146-52.

12. Waters DD, Brotons C, Chiang CW, et al. Lipid treatment assessment project 2: a multinational survey to evaluate the proportion of patients achieving low-density lipoprotein cholesterol goals. Circulation 2009; 120: 28-34.

13. Mikhailidis DP, Wierzbicki AS, Daskalopoulou SS, et al. The use of ezetimibe in achieving low density lipoprotein lowering goals in clinical practice: position statement of a United Kingdom consensus panel. Curr Med Res Opin 2005; 21: 959-69.

14. Davidson M, McGarry T, Bettis R, et al. Ezetimibe coadminis- tered with simvastatin in patients with primary hypercholesterolemia. J Am Coll Cardiol 2002; 40: 2125-34.

15. Mintz GS, Nissen SE, Anderson WD, et al. American College of Cardiology Clinical Expert Consensus Document on Standards for Acquisition, Measurement and Reporting of Intravascular Ultrasound Studies (IVUS). A Report of the American College of Cardiology Task Force on clinical Expert Consensus Documents. J Am Coll Cardiol 2001; 37: 1478-92. (Review)

16. Tani S, Watanabe I, Anazawa T, et al. Effect of pravastatin on malondialdehyde-modified low-density lipoprotein levels and coronary plaque regression as determined by three-dimensional intravascular ultrasound. Am J Cardiol 2005; 96: 1089-94.

17. Berneis K, Rizzo M, Berthold HK, Spinas GA, Krone W, GouniBerthold I. Ezetimibe alone or in combination with simvastatin increases small dense low-density lipoproteins in healthy men: a randomized trial. Eur Heart J 2010; 31: 1633-9.

18. Florentin M, Liberopoulos EN, Moutzouri E, Rizos C V, Tselepis $\mathrm{AD}$, Elisaf MS. The effect of simvastatin alone versus simvastatin plus ezetimibe on the concentration of small dense low-density lipoprotein cholesterol in subjects with primary hypercholesterolemia. Curr Med Res Opin 2011; 27: 685-92.

19. Rizzo M, Berneis K. Low-density lipoprotein size and cardiovascular risk assessment. QJM 2006; 99: 1-14. (Review)

20. Executive Summary of The Third Report of The National Cholesterol Education Program (NCEP) Expert Panel on Detection, Evaluation, and Treatment of High Blood Cholesterol in Adults (Adult Treatment Panel III). JAMA 2001; 285: 2486-97.

21. Kastelein JJ, van der Steeg WA, Holme I, et al. Lipids, apolipoproteins, and their ratios in relation to cardiovascular events with statin treatment. Circulation 2008; 117: 3002-9.

22. Kastelein JJ, Akdim F, Stroes ES, et al. Simvastatin with or without ezetimibe in familial hypercholesterolemia. N Engl J Med 2008; 358: 1431-43.

23. Fleg JL, Mete M, Howard BV, et al. Effect of statins alone versus statins plus ezetimibe on carotid atherosclerosis in type 2 diabetes diabetes: the SANDS (Stop Atherosclerosis in Native Diabetics Study) trial. J Am Coll Cardiol 2008; 52: 2198-205.

24. Meaney A, Ceballos G, Asbun J, et al. The VYtorin on Carotid intima-media thickness and overall arterial rigidity (VYCTOR) study. J Clin Pharmacol 2009; 49: 838-47.

25. Habara M, Nasu K, Terashima M, et al. Impact on optical coherence tomographic coronary findings of fluvastatin alone versus fluvastatin + ezetimibe. Am J Cardiol 2014; 113: 580-7.

26. Bogiatzi C, Spence JD. Ezetimibe and regression of carotid atherosclerosis: importance of measuring plaque burden. Stroke 2012; 43: 1153-5.

27. Nakajima N, Miyauchi K, Yokoyama T, et al. Effect of combination of ezetimibe and a statin on coronary plaque regression in patients with acute coronary syndrome: ZEUS trial (eZEtimibe Ultrasound Study). IJC Metab Endocr 2014; 3: 8-13.

28. Armitage J. The safety of statins in clinical practice. Lancet 2007 ; 370: 1781-90. (Review)

29. Preiss D, Seshasai SRK, Welsh P, et al. Risk of incident diabetes with intensive-dose compared with moderate-dose statin therapy: a meta-analysis. JAMA 2011; 305: 2556-64. 